\title{
Neoplasms of the Ampulla of Vater with Concurrent Pancreatic Intraductal Neoplasia: A Histological and Molecular Study
}

\author{
S. Nicholas Agoff, M.D., David A. Crispin, B.A., Mary P. Bronner, M.D., David H. Dail, M.D., \\ Stephen E. Hawes, M.S., Rodger C. Haggitt, M.D. \\ From the Departments of Pathology, University of Washington Medical Center/Harborview Medical Center \\ (SNA, DAC, MPB, SEH, RCH); and Virginia Mason Medical Center (DHD), Seattle, Washington
}

Adenoma and adenocarcinoma of the ampulla of Vater are uncommon neoplasms of the gastrointestinal tract. Only one report has analyzed the relationship between ampullary adenocarcinoma and pancreatic intraductal neoplasia (PanIN), the precursor lesion of pancreatic adenocarcinoma. An association between PanIN and ampullary adenoma has not been reported previously. Case reports have documented the progression of PanIN to invasive pancreatic adenocarcinoma. We reviewed five resected ampullary adenoma and 17 ampullary adenocarcinoma cases and evaluated the pancreas for PanIN. Pancreatic sections from 35 autopsies were reviewed as a control group. Immunohistochemistry for overexpression of $\mathbf{p 5 3}$ and COX-2 proteins was performed in selected cases, as was PCR analysis for K-ras mutations. Follow-up clinical data were obtained. All 22 ampullary neoplasms were associated with PanIN, which was high grade in two $(40 \%)$ adenoma cases and seven $(41 \%)$ adenocarcinoma cases. In 16 (73\%) evaluable cases, PanIN extended to the pancreatic resection margin; two of which had high grade PanIN. Among the autopsy controls eight (23\%) had low-grade PanIN. Seven of the 22 ampullary cases but none of the autopsy controls had coexistent pancreatitis. A smoking history was present in two of four autopsy cases in which this history was available. Overexpression of the p53 and COX-2 proteins was present in only one case of high-grade PanIN. K-ras mutations were present in four of four of the PanIN lesions evaluated, including one autopsy case. Clinical follow-up revealed no progression of PanIN to invasive carci-

Copyright () 2001 by The United States and Canadian Academy of Pathology, Inc.

VOL. 14, NO. 3, P. 139, 2001 Printed in the U.S.A.

Date of acceptance: October 11, 2000.

Address reprint requests to: S. Nicholas Agoff, M.D., UWMC/Harborview Medical Center, Department of Cytopathology, Box 359791, 325 Ninth Avenue, Seattle, WA 98104-2499; e-mail: agoff@u.washington.edu; fax: 206-731-8240. noma in the remnant pancreas, although the follow-up period was too short to adequately assess that risk (an average of $3.8 \mathrm{y}$ for adenoma cases and $2.5 \mathrm{y}$ for adenocarcinoma cases). We conclude that adenomas and carcinomas of the ampulla are associated with PanIN, and often high-grade PanIN. Although its malignant potential has not been fully established, PanIN is underreported and often unrecognized. PanIN may be analogous to colorectal adenoma in that both are prevalent in the older adult population, but few progress to carcinoma.

KEY WORDS: Ampullary neoplasms, Intraductal neoplasia, Pancreas, PanIN.

Mod Pathol 2001;14(3):139-146

Adenoma and adenocarcinoma of the ampulla of Vater are uncommon neoplasms of the gastrointestinal tract that are found with increased frequency in familial adenomatous polyposis syndrome (1-3). Ampullary adenoma represents the precursor lesion to adenocarcinoma, and a coexistent adenoma can be identified frequently in patients with ampullary adenocarcinoma $(1,2)$. Because of their premalignant nature, ampullary adenomas are often resected either by polypectomy or, in the case of larger lesions, by pancreaticoduodenectomy $(1,4$, 5). An association between adenomas of the ampullary region and pancreatic intraductal neoplasia has not been documented previously.

Adenocarcinoma of the pancreas is often associated with pancreatic intraductal neoplasia (PanIN), which is thought to represent the precursor lesion for this cancer (6-12). Several case reports documenting progression of high-grade (HG) PanIN to invasive pancreatic adenocarcinoma over time (17 mo to 29 y) support the role of PanIN as a precursor of carcinoma $(10,13,14)$.

The relationship between ampullary adenoma and PanIN has not been investigated previously, and only one analysis of the relationship between 
ampullary adenocarcinoma and PanIN has been reported (15). We reviewed five resected ampullary adenoma and 17 ampullary adenocarcinoma specimens and evaluated the accompanying pancreas for PanIN. Immunocytochemical and molecular analysis of the dysplastic epithelium was performed, and follow-up clinical data were obtained. In addition, pancreatic sections from 35 autopsies were examined histologically, and two were examined by molecular analysis for comparison.

\section{METHODS}

The files of the Departments of Pathology at the University of Washington Medical Center and Virginia Mason Medical Center were searched for cases of ampullary adenoma or adenocarcinoma over a 15-year period (1984-1999). The slides were reviewed by two pathologists (SNA, RCH) who confirmed the primary diagnosis and evaluated the accompanying pancreas for PanIN.

The PanIN was graded according to previously published criteria as low-grade (LG; flat epithelial hyperplasia and papillary hyperplasia without atypia) or HG (papillary epithelial hyperplasia with atypia and carcinoma in situ) PanIN (10-12). LG PanIN (Fig. 1, A-B) was defined as enlargement of the pancreatic ductal epithelial cells with abundant

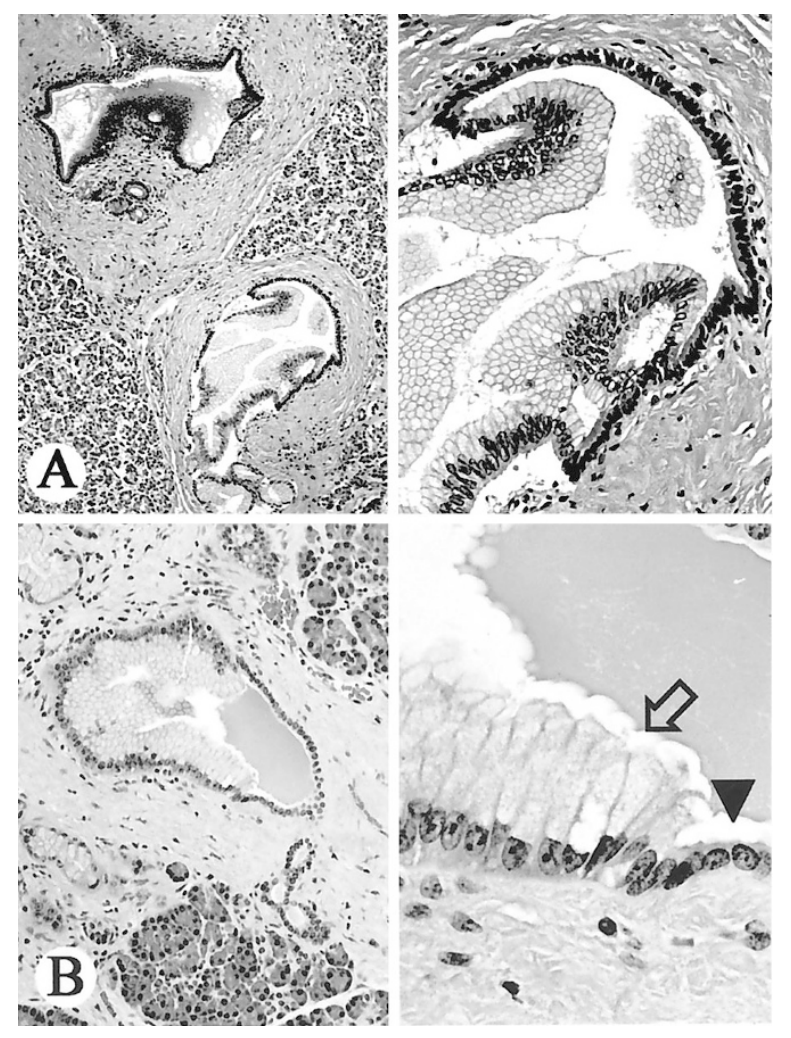

FIGURE 1. Low-grade PanIN in the pancreatic duct in (A) an autopsy control case without ampullary neoplasia and (B) in association with a tubular adenoma of the ampulla. Note the transition from the normal (arrowhead) to the neoplastic (arrow) epithelium. apical mucin and mild nuclear enlargement with variably prominent nucleoli. The neoplastic epithelium was flat or papillary with variable nuclear stratification; however, nuclear atypia was not marked, and the nuclei maintained their basal orientation and polarity, whereby the long axis of the nuclei remained perpendicular to the epithelial basement membrane. The defining feature of $\mathrm{HG}$ intraductal neoplasia (Figs. 2, 3) was marked nuclear atypia with enlarged, hyperchromatic nuclei that lost their basal orientation and no longer maintained nuclear polarity. There were usually prominent nucleoli as well as mitotic figures in the neoplastic epithelium.

The pancreatic margin was assessed for the presence and grade of PanIN, and the bile duct margin was assessed for intraductal neoplasia of the biliary epithelium. The presence of a precursor adenoma in the adenocarcinoma cases was noted, as was the presence of pancreatitis. The number of sections containing pancreatic tissue was noted. Clinical follow-up was obtained from clinical charts or directly from the primary care physician. A history of stent placement was noted when available. Intraductal papillary mucinous tumors were excluded, as were duodenal adenomas occurring more than 3 $\mathrm{cm}$ from the ampulla of Vater. There was no history of familial adenomatous polyposis in any of the patients.

Adult autopsy cases were selected at random with the following criteria: minimal autolysis of pancreatic ductal epithelium, no history of pancreatic or ampullary adenocarcinoma, and no history of familial adenomatous polyposis. Intraductal pancreatic and biliary neoplasia, when present, were graded as above. The number of sections containing pancreatic tissue was noted. Smoking history was noted when available.

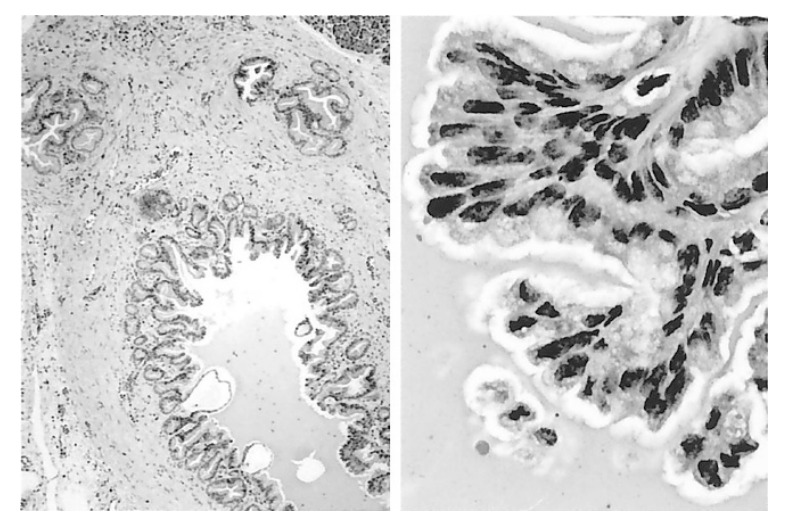

FIGURE 2. High-grade pancreatic intraductal neoplasia (PanIN) of the pancreatic duct in association with a villous adenoma of the ampulla of Vater. 


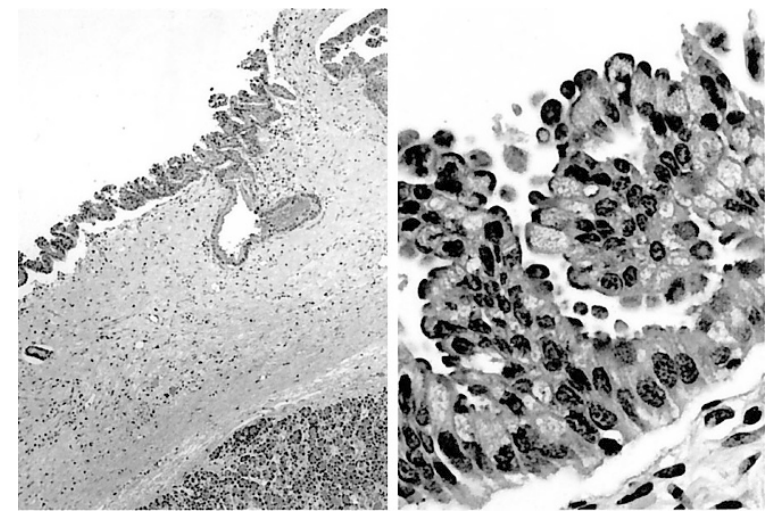

FIGURE 3. High-grade intraductal neoplasia of the common bile duct in association with a tubular adenoma of the ampulla of Vater.

\section{Immunocytochemistry}

Deparaffinized sections were immunostained with monoclonal antibodies to p53 (DAKO, Carpinteria, CA; 1:1000) and COX-2 (Cayman Chemical Company, Ann Arbor, MI; 1:1000). Negative controls consisted of substitutions of mouse ascites fluid for the primary antibody. Sections were subjected to heat-induced epitope retrieval using a microwave oven. Antigens were localized using the avidin-biotin method with 3,3'-diaminobenzidine as a chromogen, with nickel chloride enhancement. The staining was then assessed by two independent observers and graded as positive or negative according to previously published criteria (16-18). The COX-2 staining was reported as positive if the staining was +1 or greater. Internal positive controls for COX-2 were provided by mononuclear inflammatory cells.

\section{K-ras Analysis}

Cases in which PanIN lesions or normal ductal epithelium (in one autopsy control) could be easily manually microdissected were selected for this assay. Five-micrometer unstained sections of pancreas containing ducts with PanIN were subject to microdissection of the neoplastic epithelium. Genomic DNA was isolated from four pancreatic sections with PanIN and from one normal pancreatic duct from an autopsy case without PanIN.
K-ras exon 1 was amplified from genomic DNA using a thermal cycler (MJ Research, Watertown, MA) and primers as described by Imai et al. (19). Amplifications were carried out in a buffer containing $10 \mathrm{~mm}$ Tris- $\mathrm{HCl}$; $1.5 \mathrm{~mm} \mathrm{MgCl}_{2}$; and $50 \mathrm{~mm} \mathrm{KCl}$, $\mathrm{pH}$ 8.3, with $2 \mathrm{U}$ of Taq DNA polymerase (Boehringer Mannheim, Indianapolis, IN), $200 \mu \mathrm{M}$ dNTPs, and 15 pmol of each primer in a total volume of 50 $\mu \mathrm{L}$. Reactions were denatured for 3 minutes at $95^{\circ} \mathrm{C}$, then followed by 37 cycles with the following profile: $95^{\circ} \mathrm{C}(20 \mathrm{~s}), 55^{\circ} \mathrm{C}(60 \mathrm{~s})$, and $72^{\circ} \mathrm{C}(40 \mathrm{~s})$. The $\mathrm{K}$-ras mutational status of the 110-bp PCR product was assessed by denaturing gradient gel electrophoresis run at $56^{\circ} \mathrm{C}$ for 4 to 5 hours at $150 \mathrm{~V}$, then visualized using Sybr Green (Molecular Probes, Eugene, OR) staining.

\section{Statistical Analysis}

Categorical variables were compared using Pearson $\chi^{2}$ tests, or with Fisher's exact tests when sizes were small. Continuous variables, such as age, were compared using $t$ tests or analysis of variance (ANOVA), when appropriate. Logistic regression analysis was conducted to assess the association between continuous factors, such as age, and dichotomous outcomes, such as grade of disease. In all analyses, $P$ values $\leq .05$ were considered statistically significant.

\section{RESULTS}

All ampullary neoplasms, both benign and malignant, had PanIN in the pancreatic ducts, and in all but two cases, dysplasia was present in the common bile duct as well (Tables 1-3). Small, medium, and large pancreatic ducts were affected. Intraductal neoplasia was present in ducts both adjacent to the ampullary neoplasm and further away (more than $1 \mathrm{~cm}$ ).

The pancreatic ductal epithelium in two of the five adenoma cases (Fig. 4), showed HG intraductal neoplasia (Figs. 2, 3), and three had LG PanIN (Fig. 1b). In the 17 ampullary adenocarcinoma cases, the pancreatic ductal epithelium had HG PanIN in 7 and LG in 10. All of the adenoma and adenocarci-

TABLE 1. Characteristics of Patients with Periampullary Adenomas with Concurrent Pancreatic Intraductal Neoplasia (PanIN)

\begin{tabular}{|c|c|c|c|c|c|}
\hline Case & Age (y)/Sex & Adenoma & Grade of PanIN & $\begin{array}{l}\text { Bile Duct (BD) and/or } \\
\text { Pancreatic (PN) Margin } \\
\text { with Ductal Neoplasia* }\end{array}$ & Pancreatitis \\
\hline 1 & $78 \mathrm{~F}$ & Tubulovillous & Low & $\mathrm{N} / \mathrm{S}$ & No \\
\hline 2 & $39 \mathrm{M}$ & Villous & High & BD with HG; PN N/S & No \\
\hline 3 & $66 \mathrm{~F}$ & Villous & Low & $\mathrm{PN}$ negative; $\mathrm{BD}$ N/S & No \\
\hline 4 & $45 \mathrm{M}$ & Tubular & Low & $\mathrm{BD}$ and $\mathrm{PN}$ with $\mathrm{LG}$ & No \\
\hline 5 & $80 \mathrm{M}$ & Tubular & High & $\mathrm{N} / \mathrm{S}$ & Yes \\
\hline
\end{tabular}

* N/S indicates that the pancreatic and/or bile duct margin was not separately identified in the original surgical pathology report. 
TABLE 2. Characteristics of Patients with Periampullary Adenocarcinoma with Associated Adenomas and with Concurrent Pancreatic Intraductal Neoplasia (PanIN)

\begin{tabular}{|c|c|c|c|c|c|c|}
\hline Case & Age (y)/Sex & Carcinoma & $\begin{array}{c}\text { Associated } \\
\text { Adenoma }\end{array}$ & Grade of PanIN & $\begin{array}{c}\text { Neoplasia Involving } \\
\text { the Bile Duct (BD) } \\
\text { or Pancreatic (PN) } \\
\text { Margin* }\end{array}$ & Pancreatitis \\
\hline 1 & $49 / \mathrm{M}$ & Intramucosal & Villous & High (focal) & PN and BD with LG & No \\
\hline 2 & $58 / \mathrm{F}$ & Well differentiated & Villous & Low & $\mathrm{BD}$ and $\mathrm{PN}$ negative & No \\
\hline 3 & $75 / \mathrm{F}$ & Well differentiated & Villous & Low & PN with LG & Yes \\
\hline 4 & $61 / \mathrm{M}$ & Mod. differentiated & Tubulovillous & High (focal) & $\begin{array}{l}\text { PN with HG } \\
\text { BD with LG }\end{array}$ & No \\
\hline 5 & $76 / \mathrm{F}$ & Mod. differentiated & Tubular & Low & PN and BD with LG & No \\
\hline 6 & $77 / \mathrm{M}$ & Mod. differentiated & Villous & High (focal) & PN and BD with LG & Yes \\
\hline 7 & $72 / \mathrm{M}$ & Mod. differentiated & Villous & High (focal) & $\mathrm{BD}$ and $\mathrm{PN}$ negative & No \\
\hline 8 & $66 / \mathrm{F}$ & Mod. differentiated & Tubular & Low & BD with LG & No \\
\hline 9 & $63 / \mathrm{M}$ & Mod. differentiated & Tubulovillous & Low & $\mathrm{BD}$ and $\mathrm{PN} N / \mathrm{S}$ & No \\
\hline 10 & $65 / F$ & Mod. differentiated & Tubulovillous & Low & PN and BD with LG & Yes \\
\hline 11 & $78 / \mathrm{F}$ & Poorly differentiated & Villous & Low & BD with LG & No \\
\hline 12 & $27 / \mathrm{M}$ & Poorly differentiated & Tubulovillous & Low & PN and BD with LG & No \\
\hline 13 & $69 / \mathrm{M}$ & Poorly differentiated & Villous & High & $\begin{array}{l}\text { BD with LG } \\
\mathrm{PN} \text { N/S }\end{array}$ & No \\
\hline 14 & $75 / \mathrm{M}$ & Poorly differentiated & Tubulovillous & Low & PN and BD with LG & Yes \\
\hline 15 & $61 / \mathrm{F}$ & Poorly differentiated & Villous & High (focal) & PN with LG & Yes \\
\hline 16 & $51 / \mathrm{M}$ & Poorly differentiated & Villous & High & PN with LG & Yes \\
\hline 17 & $62 / \mathrm{M}$ & Poorly differentiated & Tubular & Low & BD with LG & No \\
\hline
\end{tabular}

${ }^{*} \mathrm{~N} / \mathrm{S}$ indicates that the pancreatic and/or bile duct margins were not separately identified in the original surgical pathology report.

noma cases with LG PanIN had papillary hyperplasia without atypia, except for one carcinoma case (Carcinoma 8, Table 2), which had only flat epithelial hyperplasia. Sixteen of the 22 cases had intraductal neoplasia extending to either the pancreatic or bile duct margins, two of which were HG (Adenoma 2, Table 1 and Carcinoma 4, Table 2). In six cases, the original surgical pathology report did not specifically identify the pancreatic and/or bile duct margins, and they therefore could not be evaluated.

The average age in the adenoma group was 62 years (median, $66 \mathrm{y}$; range, 45 to $80 \mathrm{y}$ ), and in the carcinoma group, 64 y (median, $65 \mathrm{y}$; range, 27 to $78 \mathrm{y})$, which was not a significant difference $(P=$ .8). The average age of patients with HG PanIN was 60 years in the adenoma group (median, $60 \mathrm{y}$; range, 39 to $80 \mathrm{y}$ ) and 63 years in the carcinoma group (median, $61 \mathrm{y}$; range, 49 to $77 \mathrm{y}$ ). The maleto-female ratio was 1.6:1. Seven of the 22 cases had coexistent pancreatitis, and there was a history of stent placement in only two of the cases (one adenoma and one carcinoma case that were not associated with HG PanIN).

The pancreatic ductal epithelium in $8(23 \%)$ of the 35 autopsy controls revealed LG PanIN: six with flat epithelial hyperplasia and two with papillary hyperplasia without atypia (Fig. 1a). None of the autopsy controls had HG PanIN. The average age for the 35 controls was $68 \mathrm{y}$ (median, $69 \mathrm{y}$; range, 37 to $90 \mathrm{y}$ ), and the average age of those with PanIN was also $68 \mathrm{y}$ (median, $66 \mathrm{y}$; range, 40 to $90 \mathrm{y}$ ). The male-to-female ratio was $1.7: 1$. Of the four patients for whom a smoking history was available, two were smokers, and two were nonsmokers. There was no evidence of pancreatitis in any of the autopsy cases.
PanIN was significantly more prevalent in the adenoma/adenocarcinoma cases compared with the case of controls $(P<.001)$. However, the ampullary adenoma/adenocarcinoma cases had significantly more sections of pancreas submitted for evaluation than did the autopsy cases $(P<.05)$. The adenoma cases had an average of 6.4 sections of pancreatic tissue (median of 5, range of 3-13); the adenocarcinoma cases had an average of $5.5 \mathrm{sec}-$ tions (median, 6.5; range, 3-10); and the autopsy cases, an average of 1.1 sections (median, 1; range, $1-2)$.

There was no significant association between the age of the patients and the grade of intraductal neoplasia $(P=.7)$. However, male gender was significantly associated with the grade of PanIN $(P=$ $.02)$; only 5 of 13 cases with LG, versus 8 of 9 cases with HG neoplasia, were in males. In both the ampullary adenoma and adenocarcinoma cases, we observed a male predominance in cases with HG PanIN. There was no significant association between pancreatitis and the presence of intraductal

TABLE 3. Characteristics of Autopsy Controls with Pancreatic Intraductal Neoplasia (PanIN; 8 of 35 Cases)

\begin{tabular}{cclclc}
\hline Case & Age $(\mathrm{y})$ & Sex & $\begin{array}{l}\text { PanIN } \\
\text { Grade }\end{array}$ & $\begin{array}{l}\text { Smoking } \\
\text { History* }\end{array}$ & Pancreatitis \\
\hline 1 & 90 & Female & Low & N/A & No \\
2 & 57 & Male & Low & Positive & No \\
3 & 87 & Female & Low & N/A & No \\
4 & 86 & Male & Low & N/A & No \\
5 & 74 & Male & Low & Positive & No \\
6 & 40 & Male & Low & N/A & No \\
7 & 55 & Male & Low & Negative & No \\
8 & 53 & Female & Low & Negative & No \\
\hline
\end{tabular}

* N/A indicates that smoking history was not available. 


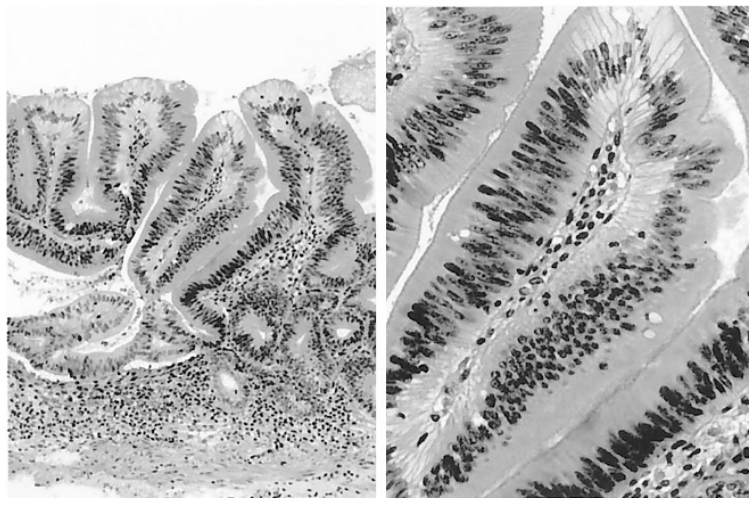

FIGURE 4. Villous adenoma of the ampulla of Vater.

neoplasia $(P=.09)$ in the cases and controls and no significant association between pancreatitis and the grade of PanIN in the cases $(P=.3)$.

Immunohistochemical analysis of p53 protein in the adenoma and adenocarcinoma cases revealed overexpression of p53 in only one focus of HG PanIN in the eight cases evaluated, whereas the associated ampullary neoplasms had overexpression in five of the eight cases (three adenomas, two adenocarcinoma; Table 4). One case (Carcinoma 17, Table 4) had overexpression of p53 in the adenoma but not in the carcinoma. COX-2 was overexpressed in only one case of HG PanIN, the associated adenocarcinoma, and in one other adenocarcinoma. All adenomas were negative for COX-2 protein overexpression. The two autopsy cases evaluated, one with LG PanIN and one with no intraductal neoplasia, had no overexpression of p53 or COX-2.

PCR analysis of $\mathrm{K}$-ras revealed point mutations at Codon 12 in all of the PanIN lesions evaluated (four of four), including one autopsy control case (Table 4). The one autopsy pancreas control case without PanIN showed no mutation of K-ras.

Clinical follow-up of the adenoma cases revealed that all five patients were alive and well, with an average follow-up interval of 3.8 years (range, 0.3 to
8.8 y); the one patient with HG PanIN at the resection margin is currently disease free at 8.0 years. Of the adenocarcinoma cases, three $(23 \%)$ were dead with recurrent disease, two (15\%) were dead with unknown cancer status, and eight (62\%) were alive and well. The average follow-up period was 2.5 years (range, 0.3 to $8.8 \mathrm{y}$ ). A carcinoma patient with HG PanIN at the resection margin is currently disease free at 2 years. For three patients, follow-up information was not available, and one case was too recent for follow-up.

\section{DISCUSSION}

The link between pancreatic adenocarcinoma and the precursor lesion of pancreatic intraductal neoplasia (PanIN) was first suggested by Sommers and coworkers (20). Since then, many studies have shown an association between pancreatic adenocarcinoma and intraductal neoplasia $(6,7,9)$. PanIN has been estimated to occur commonly in patients without a history of pancreatic cancer in autopsy samples and in pancreatic tissue removed for nonneoplastic disease, with different studies having a prevalence of around 20 to $30 \%$, though it is considerably more frequent in patients with carcinoma $(6,7,8,21)$. High-grade PanIN occurs only rarely in patients without a history of pancreatic neoplasia $(6,7)$.

Previous studies have shown some relationship between PanIN and increasing age, smoking history, chronic pancreatitis, and the number of sections of pancreatic tissue analyzed $(6,12,21-28)$. We did not find a significant association between age and dysplasia, but we did find a significant association of HG PanIN with male gender, though our study is limited. Only two of the four autopsy cases with PanIN in the current study had a smoking history, indicating that it occurs in nonsmokers; more recent studies have not shown a significant association with smoking (28). (We did not assess the smoking history in ampullary neoplasia cases;

TABLE 4. p53 and COX-2 Immunohistochemistry Results for the Ampullary Neoplasm and the Intraductal Neoplastic Epithelium; K-ras PCR Results on the Microdissected Intraductal Neoplastic Epithelium (PanIN)

\begin{tabular}{lll}
\hline \multicolumn{1}{c}{ Case* } & \multicolumn{1}{c}{ p53 } & \multicolumn{1}{c}{ K-ras** } \\
\hline Adenoma-2 & Negative in adenoma/negative in HG PanIN & Negative in adenoma/negative in HG PanIN \\
Adenoma-3 & Positive in adenoma/negative in LG PanIN & Negative in adenoma/negative in LG PanIN \\
Adenoma-4 & Positive in adenoma/negative in LG PanIN & Negative in adenoma/negative in LG PanIN \\
Adenoma-5 & Negative in HG PanIN/negative in adenoma & Negative in HG PanIN/negative in adenoma \\
Carcinoma-1 & Negative in HG PanIN/negative in carcinoma & Negative in HG PanIN/negative in carcinoma $\quad$ Positive \\
Carcinoma-10 & Positive in carcinoma/negative in LG PanIN & Negative in carcinoma/negative in LG PanIN $\quad$ ND \\
Carcinoma-11 & Positive in carcinoma/negative in LG PanIN & Negative in carcinoma/negative in LG PanIN \\
Carcinoma-13 & Negative in carcinoma/positive in HG PanIN & Positive in carcinoma/positive in HG PanIN $\quad$ Positive \\
Carcinoma-17 & Focally positive in adenoma/negative in LG PanIN & Positive in carcinoma/negative in LG PanIN \\
Autopsy-1 & Negative in LG PanIN & Negative in LG PanIN \\
Autopsy (no PanIN) & Negative in epithelium & Negative in epithelium \\
\hline
\end{tabular}

PanIN, pancreatic intraductal neoplasia.

* Numbers refer to case numbers in Tables 1-3.

** Positive indicates that a mutation was present in Codon 12 of the c-K-ras gene. ND indicates that k-ras PCR was not done in the specified case. 
we were interested only in exploring the prevalence of PanIN in association with ampullary neoplasia and not the epidemiologic factors with which it may be associated.) As a surrogate marker for duct obstruction, all of our cases were assessed for the presence of pancreatitis, and there was no significant association in our limited study between the presence of pancreatitis and PanIN or between pancreatitis and the grade of PanIN. The adenoma/ adenocarcinoma cases had significantly more sections of pancreatic tissue submitted than the autopsy controls, which may account for some of the increased frequency of PanIN detection in these cases.

Adenomas of the ampullary region have not previously been reported to be associated with PanIN. We found that ampullary adenomas not only are associated with PanIN but often with HG PanIN. In $40 \%$ of our adenoma cases, these intraductal lesions were not mentioned in the surgical pathology report, and the status of the pancreatic and biliary margins was sometimes not specified.

Only one previous report has analyzed the relationship between PanIN and ampullary adenocarcinomas (15). Liu and coworkers found intraductal neoplasia in the large pancreatic ducts in $21.9 \%$ of cases of ampullary carcinoma; however, they excluded cases of flat epithelial hyperplasia. All of the adenocarcinoma cases in the present study that had LG PanIN had papillary epithelial hyperplasia without atypia except for one case, which had flat epithelial hyperplasia. As above, these lesions were often overlooked in the original surgical pathology evaluation in $59 \%$ of the cases, although margins were more often specified than for the adenoma cases.

Surgical resection of ampullary adenomas is associated with low morbidity and mortality, and ampullary adenocarcinoma is more often resectable and has an increased survival time relative to pancreatic adenocarcinoma (2, 5, 29). Typical 5-year survival rates for ampullary adenocarcinoma after pancreaticoduodenectomy are around $60 \%$, compared with the dismal 5-year survival rate of around $3.5 \%$ for carcinomas of pancreatic ductal origin (29, 30 ). The patients with ampullary adenoma in this study were all alive and well, with an average follow-up period of 3.8 years, and $62 \%$ of patients with ampullary adenocarcinoma were alive and well with a 2.5-year average postoperative follow-up period, figures that are comparable to published survival rates for ampullary adenocarcinoma.

In the past, PanIN was divided into four categories: flat epithelial hyperplasia, papillary epithelial hyperplasia, papillary hyperplasia with atypia, and carcinoma in situ $(6,9)$. Recently, because of the unknown neoplastic potential of flat and papillary epithelial hyperplasia and the poor reproducibility among pathologists in separating papillary hyperplasia with atypia and carcinoma in situ, a new grading system was proposed: LG PanIN (flat and papillary epithelial hyperplasia) and HG PanIN (papillary hyperplasia with atypia and carcinoma in situ; 10-12). These terms are equivalent to LG and HG pancreatic ductal dysplasia and are thought to separate the lesions into those with a low probability for neoplastic progression (LG PanIN) and a high probability for progression (HG PanIN; 11, 12, 21), though this grading scheme is not yet widely accepted.

The neoplastic nature of LG and HG PanIN is supported by the discovery of multiple genetic alterations within it, most notably K-ras mutation, overexpression of p53 protein, and loss of p16 protein expression (31-34). K-ras abnormalities, most often mutations in Codon 12, have been demonstrated in neoplastic ductal epithelium isolated from patients with a history of pancreatic cancer as well as from those with no history of pancreatic neoplasia and from autopsy specimens (33-36). Overexpression of p53 is found most commonly in invasive pancreatic carcinoma; however, it may also be overexpressed in PanIN, especially HG PanIN (34, 37-39). Apple and colleagues (34) demonstrated a relationship between p53 overexpression and increasing grades of epithelial atypia. They suggested that p53 alterations may occur later in pancreatic carcinogenesis as they are found more commonly in HG lesions and invasive carcinoma (34).

To confirm that the lesions we were interpreting as PanIN were, in fact, neoplastic, we carried out K-ras mutational analysis and immunohistochemical assays for p53 and COX-2 protein overexpression on some of them. The intraductal neoplastic lesions we analyzed for a K-ras mutation, one HG and three LG PanIN, showed mutations of K-ras at Codon 12, including the autopsy case. The autopsy control with normal-appearing epithelium showed no mutation of K-ras. Overexpression of p53 was present in one case of HG PanIN, which also had a mutation in K-ras (Carcinoma 13, Table 4). Interestingly, this case showed no overexpression of p53 in the associated carcinoma. None of the LG intraductal lesions had overexpression of p53.

Cyclooxygenase-2 (COX-2) has been shown to play an important and early role in colorectal neoplasia, and overexpression of COX-2 has been found in approximately $72 \%$ of pancreatic adenocarcinomas $(18,40,41)$. We found overexpression of COX-2 in two of the five ampullary adenocarcinomas evaluated and overexpression in one associated focus of HG PanIN. This case was the same one that showed overexpression of p53 protein in the neoplastic ductal epithelium (Carcinoma 13, Table 4). The ampullary adenoma and other cases of Pa- 
nIN were negative for COX-2 overexpression. This implies that overexpression of COX-2 may play a later role in ampullary neoplasia and possibly in pancreatic ductal neoplasia than it does in colorectal neoplasia, since normal colonic epithelium near neoplastic lesions, as well as most adenomatous lesions of the colon, show COX-2 overexpression (18).

The importance of PanIN in patients with ampullary neoplasms is uncertain. Patients with ampullary neoplasms, including ampullary adenocarcinoma, have a relatively favorable prognosis with long survival times, which could increase the probability of progression of the PanIN lesions. Nordback and colleagues reported two patients with ampullary carcinoma who developed a second primary adenocarcinoma 5 and 15 years after resection of the ampullary lesions (13). The initial resection specimens in both patients had dysplasia in the hepatic duct. Other studies report the progression of HG PanIN to invasive carcinoma 17 months to 29 years after diagnosis $(10,14)$. This implies that progression of PanIN in patients with ampullary neoplasms is possible, especially in view of their long survival times.

The follow-up period in our study was too short to determine whether pancreatic or biliary ductal carcinomas will develop; it averaged 3.8 years (range, 0.3 to $8.8 \mathrm{y}$ ) for the adenoma cases and 2.5 years (range, 0.3 to 8.8 y) for the carcinoma cases. Two cases had HG PanIN extending to the resection margin, and both of those patients are currently disease free at 8 and 2 years, respectively. However, according to the previously cited reports, it may take as long as 29 years for even HG PanIN to progress to invasive carcinoma. Regular follow-up of these patients should be considered.

Although we show that ampullary adenomas and adenocarcinomas are associated with PanIN, the exact nature of this association is unknown. The one case mentioned previously that showed p53 overexpression in the HG PanIN but not in the associated carcinoma (Carcinoma 13, Table 4) suggests that these lesions may not be directly related, though drawing a conclusion from one case is a tenuous proposition at best. Whether this association could be due to a field effect similar to that seen in the urinary bladder is also speculative, and further investigation is required to determine the nature of this association.

The natural history of LG PanIN remains to be elucidated. An analogy has been drawn between the adenoma-carcinoma sequence in the colon and PanIN in the pancreas (12). Colonic adenomas are common, and the number of patients who have them increases with age, yet only a small number progress to colonic adenocarcinoma (42). Perhaps LG PanIN is analogous to tubular adenomas of the colon in that although it is common, few progress to carcinoma. This analogy can be applied to the molecular events seen in PanIN as well, with early abnormalities of K-ras present in LG lesions and later, abnormalities in p53, as seen in the colonic mucosa with neoplastic progression $(36,37,43)$.

In conclusion, both ampullary adenoma and adenocarcinoma are associated with PanIN, and often with HG PanIN. The explanation for the concurrence of ampullary neoplasms and intraductal neoplasia of the pancreatic and biliary epithelium is unknown, and further research is needed to establish whether this is a field effect similar to that seen in neoplasms of the urinary bladder or if it is due to other phenomena. The overexpression of p53 and COX-2 proteins in the intraductal lesions in patients with ampullary neoplasms, and the K-ras mutations in both the autopsy controls and the patients, provides support for the neoplastic nature of PanIN. The fact that PanIN was often not mentioned in the surgical pathology reports, even when it was present at a surgical margin, emphasizes the need for increased recognition of these intraductal lesions. Though no progression of the PanIN lesions was present in our series, our follow-up period was too short to adequately assess the risk. Intraductal neoplasia of the pancreas may be analogous to adenomas of the colon, and further investigation is needed to establish what factor(s) are responsible for progression of these lesions.

Acknowledgment: This article is dedicated to our friend, mentor, and teacher, Dr. Rodger Haggitt. You will live in our memories forever.

\section{REFERENCES}

1. Qizilbash AH. Duodenal and peri-ampullary adenomas. Curr Top Pathol 1990;81:77-90.

2. Talbot IC, Neoptolemos JP, Shaw DE, Carr-Locke D. The histopathology and staging of carcinoma of the ampulla of Vater. Histopathology 1988;12:155-65.

3. Bertoni G, Sassatelli R, Nigrisoli E, Pennazio M, Tansini P, Arrigoni A, et al. High prevalence of adenomas and microadenomas of the duodenal papilla and periampullary region in patients with familial adenomatous polyposis. Eur J Gastroenterol Hepatol 1996;8:1201-6.

4. Bertoni G, Sassatelli R, Nigrisoli E, Bedogni G. Endoscopic snare papillectomy in patients with familial adenomatous polyposis and ampullary adenoma. Endoscopy 1997;29:685-8.

5. Treitschke F, Beger HG. Local resection of benign periampullary tumors. Ann Oncol 1999;10:212-4.

6. Cubilla AL, Fitzgerald PJ. Morphological lesions associated with human primary invasive nonendocrine pancreas cancer. Cancer Res 1976;36:2690-8.

7. Kozuka S, Sassa R, Taki T, et al. Relation of pancreatic duct hyperplasia to carcinoma. Cancer 1979;43:1418-28.

8. Kloppel G, Bommer G, Ruckert K, Seifert G. Intraductal proliferation in the pancreas and its relationship to human and 
experimental carcinogenesis. Virchows Arch A Pathol Anat 1980;387:221-33.

9. Chen J, Baithun SI, Ramsay MA. Histogenesis of pancreatic carcinomas: a study based on 248 cases. J Pathol 1985;146: 65-76.

10. Brat DJ, Lillemoe KD, Yeo CJ, Warfield PB, Hruban RH. Progression of pancreatic intraductal neoplasias to infiltrating adenocarcinoma of the pancreas. Am J Surg Pathol 1998; 22:163-9.

11. Klimstra DS, Longnecker DS. K-ras mutations in pancreatic ductal proliferative lesions [letter] [comment]. Am J Pathol 1994;145:1547-50.

12. Furukawa T, Chiba R, Kobari M, Matsuno S, Nagura $H$, Takahashi T. Varying grades of epithelial atypia in the pancreatic ducts of humans. Classification based on morphometry and multivariate analysis and correlated with positive reactions of carcinoembryonic antigen. Arch Pathol Lab Med 1994;118:227-34.

13. Nordback IH, Hruban RH, Cameron JL. Second primary lesions in the biliary tree after successful resection of ampullary carcinoma. Surgery 1992;112:111-5.

14. Brockie E, Anand A, Albores-Saavedra J. Progression of atypical ductal hyperplasia/carcinoma in situ of the pancreas to invasive adenocarcinoma. Ann Diagn Pathol 1998;2:286-92.

15. Liu TH, Chen J, Zeng XJ. Histogenesis of pancreatic head and ampullary region carcinoma. Chin Med J (Engl) 1983;96:16774.

16. Davidoff AM, Humphrey PA, Iglehart JD, Marks JR. Genetic basis for p53 overexpression in human breast cancer. Proc Natl Acad Sci U S A 1991;88:5006-10.

17. Lee CS, Pirdas A. p53 protein immunoreactivity in cancers of the gallbladder, extrahepatic bile ducts and ampulla of Vater. Pathology 1995;27:117-20.

18. Hao $\mathrm{X}$, Bishop AE, Wallace $\mathrm{M}$, et al. Early expression of cyclo-oxygenase-2 during sporadic colorectal carcinogenesis. J Pathol 1999;187:295-301.

19. Imai M, Hoshi T, Ogawa K. K-ras codon 12 mutations in biliary tract tumors detected by polymerase chain reaction denaturing gradient gel electrophoresis. Cancer 1994;73:2727-33.

20. Sommers SC, Murphy SA, Warren S. Pancreatic duct hyperplasias and cancer. Gastroenterology 1954;27:629-40.

21. Stamm BH. Incidence and diagnostic significance of minor pathologic changes in the adult pancreas at autopsy: a systematic study of 112 autopsies in patients without known pancreatic disease. Hum Pathol 1984;15:677-83.

22. Chiaramondia M, Ceppa P. [Epithelial dysplasia and stroma changes in chronic pancreatitis and carcinoma of the exocrine pancreas. Morphologico-functional correlations]. Pathologica 1988;80:561-72.

23. Cylwik B, Nowak HF, Puchalski Z, Barczyk J. [Chronic pancreatitis as a predisposing factor in the development of pancreatic cancer. Histological and histochemical studies]. Zentralbl Allg Pathol 1985;130:217-24.

24. Cylwik B, Nowak HF, Puchalski Z, Barczyk J. Epithelial anomalies in chronic pancreatitis as a risk factor of pancreatic cancer. Hepatogastroenterology 1998;45:528-32.

25. Mukada T, Yamada S. Dysplasia and carcinoma in situ of the exocrine pancreas. Tohoku J Exp Med 1982;137:115-24.

26. Kishi K, Nakamura K, Yoshimori M, Tajiri H, Ozaki H, Kinoshita T, et al. Morphology and pathological significance of focal acinar cell dysplasia of the human pancreas. Pancreas 1992;7:177-82.

27. Auerbach O, Garfinkel L. Histologic changes in pancreas in relation to smoking and coffee-drinking habits. Dig Dis Sci 1986;31:1014-20.

28. Tomioka T, Andren-Sandberg A, Fujii H, Egami H, Takiyama Y, Pour PM. Comparative histopathological findings in the pancreas of cigarette smokers and non-smokers. Cancer Lett 1990;55:121-8.

29. Shutze WP, Sack J, Aldrete JS. Long-term follow-up of 24 patients undergoing radical resection for ampullary carcinoma, 1953 to 1988. Cancer 1990;66:1717-20.

30. Gudjonsson B. Cancer of the pancreas. 50 years of surgery. Cancer 1987;60:2284-303.

31. Longnecker DS. The quest for preneoplastic lesions in the pancreas [editorial; comment]. Arch Pathol Lab Med 1994; 118:226.

32. Wilentz RE, Geradts J, Maynard R, Offerhaus GJ, Kang M, Goggins M, et al. Inactivation of the p16 (INK4A) tumorsuppressor gene in pancreatic duct lesions: loss of intranuclear expression. Cancer Res 1998;58:4740-4.

33. Moskaluk CA, Hruban RH, Kern SE. p16 and K-ras gene mutations in the intraductal precursors of human pancreatic adenocarcinoma. Cancer Res 1997;57:2140-3.

34. Apple SK, Hecht JR, Lewin DN, Jahromi SA, Grody WW, Nieberg RK. Immunohistochemical evaluation of K-ras, p53, and HER-2/neu expression in hyperplastic, dysplastic, and carcinomatous lesions of the pancreas: evidence for multistep carcinogenesis. Hum Pathol 1999;30:123-9.

35. Tada M, Ohashi M, Shiratori Y, Okudaira T, Komatsu Y, Kawabe T, et al. Analysis of K-ras gene mutation in hyperplastic duct cells of the pancreas without pancreatic disease [see comments]. Gastroenterology 1996;110:227-31.

36. Di Giuseppe JA, Hruban RH, Offerhaus GJ, Clement MJ, van den Berg FM, Cameron JL, et al. Detection of K-ras mutations in mucinous pancreatic duct hyperplasia from a patient with a family history of pancreatic carcinoma [see comments]. Am J Pathol 1994;144:889-95.

37. Di Giuseppe JA, Hruban RH, Goodman SN, Polak M, van den Berg FM, Allison DC, et al. Overexpression of p53 protein in adenocarcinoma of the pancreas. Am J Clin Pathol 1994;101: $684-8$.

38. Boschman CR, Stryker S, Reddy JK, Rao MS. Expression of p53 protein in precursor lesions and adenocarcinoma of human pancreas. Am J Pathol 1994;145:1291-5.

39. Zhang SY, Ruggeri B, Agarwal P, Sorling AF, Obara T, Ura H, et al. Immunohistochemical analysis of p53 expression in human pancreatic carcinomas. Arch Pathol Lab Med 1994; 118:150-4.

40. Koshiba T, Hosotani R, Miyamoto Y, Wada M, Lee JU, Fujimoto $\mathrm{K}$, et al. Immunohistochemical analysis of cyclooxygenase-2 expression in pancreatic tumors. Int J Pancreatol 1999;26:69-76.

41. Molina MA, Sitja-Arnau M, Lemoine MG, Frazier ML, Sinicrope FA. Increased cyclooxygenase-2 expression in human pancreatic carcinomas and cell lines: growth inhibition by nonsteroidal anti-inflammatory drugs. Cancer Res 1999;59: 4356-62.

42. Day DW, Morson BC. The adenoma-carcinoma sequence. Major Probl Pathol 1978;10:58-71.

43. Fearon ER, Vogelstein B. A genetic model for colorectal tumorigenesis. Cell 1990;61:759-67. 